\title{
La comptabilité nationale de l'eau en France : instrument d'analyse et de gestion patrimoniale
}

\author{
National water comptability in France : \\ an instrument for analysing and managing
}

\author{
par Pascale Babillot, \\ IFEN \\ et Jean Margat, \\ BRGM
}

Water has been counted, balanced and statistically assessed for many decades in a variety of situations ranging from a hydrologist's gauging station to the thrifty household's water meter. A new water accounting system is now proposed in order to connect and integrate all separate accounts in a macro-economic and patrimonial perspective. Why and how should we account for water? Water is a raw material : we need to measure what is offered us by nature, what we take ourselves and what is offered us by nature, what we take for ourselves and what effects we produce on the water resource. By applying methods used in national economical accounting to the new science of ecological accounting, this instrument is intended to serve analysts and forecasters involved in water policy and management.

\section{INTRODUCTION}

La comptabilité nationale et régionale de l'eau a été conçue initialement dans le cadre des comptes du patrimoine naturel promus par la Commission interministérielle ad hoc (CICPN) à partir de 1982, qui visaient à promouvoir la prise en compte de l'environnement dans la Comptabilité nationale économique. L'objectif de ces comptes de l'eau était de relier avec cohérence, suivant des références spatiales et temporelles homogènes et pertinentes, les chiffrages et les statistiques sur les ressources en eau d'une part, sur les effets des aménagements et les utilisations d'eau d'autre part, afin de permettre une comptabilité analytique et une évaluation des pressions des utilisations sur les ressources.

La première phase de ces travaux a eu surtout pour but d'élaborer et d'éprouver une méthode, en s'attachant aux comptes en quantités simples et en se bornant à explorer les voies et les modes de comptes en qualité. Une nomenclature et une structure des tableaux comptables appropriés ont été mises au point, ainsi que les démarches de collecte et de traitement des données nécessaires. Les principes et les modalités opérationnelles des comptes de l'eau ont été présentés dans la publication de l'INSEE sur les “ Comptes du patrimoine naturel " [1], notamment dans son chapitre 5 "Compte des eaux continentales".

Après un premier exercice de comptabilité régionale annuelle réalisé en 1983-84 sur le département du Calvados (divisé en 10 bassins élémentaires) pour éprouver la faisabilité de l'approche conçue, l'établissement de comptes annuels de la France entière et de chacun des 6 bassins (Agences de l'Eau) a été réalisé pour l'année 1981, sous la responsabilité du ministère de l'Environnement (Direction de la prévention des pollutions / Service de l'eau) avec le concours du BRGM, en 1985.

Entérinée par l'OCDE (Comité de l'environnement 1988, [2]) cette approche comptable française a inspiré ensuite plusieurs exercices à l'étranger : en Indonésie sur un bassin de Java [3], aux Etats-Unis sur le bassin de la rivière Weber (Utah) [4], en Espagne [5] où les comptes physiques ont été reliés à la comptabilité économique nationale.

Enfin, plus récemment, deux nouveaux exercices comptables ont été réalisés à l'initiative de l'IFEN :

- actualisation de l'évaluation de faisabilité des comptes physiques dans un cadre plus régional (le haut bassin de la Loire), et affinement des références temporelles (comptes trimestriels dans le cadre de l'année hydrologique 1989-90 $[6,7])$,

- modélisation simplifiée des comptes de qualité par bassins versants à partir de trois cartes de qualité linéaires des cours d'eau 1994 et 1996, publiées par le Réseau National de Données sur l'Eau [8].

\section{LES COMPTES DE L'EAU EN QUANTITÉ : UNE APPROCHE ORIGINALE}

Par rapport aux chiffrages et aux statistiques classiques respectives sur les ressources en eau et sur les utilisations opérées séparément en suivant des références territoriales et 


\section{ENCADRE 1}

\section{QUELQUES RÉSULTATS (EN KM³) ET INDICATEURS (EN \%) DU COMPTE DE L'EAU DE LA FRANCE ENTIÈRE}

année 1981

- Apports naturels totaux : $593 \mathrm{~km}^{3}$

(en net excédent par rapport à un année moyenne de l'ordre de 450), dont :

- précipitations : 575

- écoulements entrants : 18 (affluences nettes, Rhin frontalier exclu)

- De ce volume total :

- $262(44 \%)$ se sont écoulés à la mer ou en pays voisins,

- $22(3,7 \%)$ ont été stockés : solde positif des mouvements de stocks de neige et de nappes souterraines.

- La ressource naturelle a été de 292 : précipitations non évaporées et affluences.

- L'ensemble des utilisations a :

- régularisé 3,8 ( $1,3 \%$ de la ressource) : lâchures des retenues dans les rivières,

- prélevé 37,2 (13\% de la ressource, ou $27,5 \%$ de la ressource régulière, formée par l'apport des nappes, neiges et glaciers aux cours d'eau), dont : $15 \%$ par les producteurs d'eau potable,

$14 \%$ par les industries non desservies,

$12 \%$ par l'agriculture,

$52 \%$ par les producteurs d'énergie (thermoélectricité),

$7 \%$ par les services publics (municipalités, navigation,...)

- restitué 30 aux eaux continentales $(80,6 \%$ des prélèvements) : retours d'eaux urbains, industriels et énergétiques,

- consommé $0,66(0,23 \%$ de la ressource, ou $2 \%$ des prélèvements) par évaporation ou déversement en mer ; il faut ajouter l'évaporation induite par l'irrigation $(3,45)$ qui est intégrée dans le compte à l'évapotranspiration totale,

- épargné 0,6 : solde bénéficiaire des retenues, dont le stock total s'est accru de $6,7 \%$

- L'évaporation totale, naturelle ou induite par l'irrigation $(304,7)$ et induite par les autres usages $(0,66)$, se serait élevée à 305 , soit un peu plus de la moitié $(51 \%)$ des apports naturels totaux, équivalent à une hauteur d'eau uniforme sur tout le pays de $555 \mathrm{~mm}$.

temporelles indépendantes et généralement sans cohérence, le "plus " apporté par l'approche comptable consiste à rapprocher des données d'origines très diverses, à unifier les références spatio-temporelles pour rendre les chiffrages cohérents, à marier des comptes de stock et des comptes de flux suivant une structure budgétaire, et à mettre en correspondance les subdivisions des systèmes tant de ressources que d'utilisation pour permettre une comptabilité non seulement globale mais analytique.

A cette fin, la structure des tableaux comptables adoptés met en relation (cf les exemples des tableaux 1 et 2 du compte de la France entière en 1981, présentés ci-après sous une forme simplifiée) :

- les subdivisions des systèmes physiques de ressource en eau en " branches " macroscopiques correspondant aux types de milieu-eau (eau du sol, eaux souterraines, cours d'eau, lacs...) qui constituent des catégories de source d'approvisionnement et des sous-systèmes de gestion de ressource,

- les classes d'agents du système d'utilisation (opérateurs d'aménagement, producteurs et/ou usagers d'eau, acteurs de rejets...).

Cette structure comptable est flexible et peut être plus ou moins subdivisée ou simplifiée suivant les cas traités.

Elle impose de fortes contraintes de cohérence aux différents résultats chiffrés, ainsi qu'au jeu des divers indicateurs d'économie de l'eau ou de pression sur les ressources calculées sur leur base.
Elle permet aussi, en principe, la liaison entre les comptes d'agents physiques et les comptes économiques monétaires.

Les comptes de l'eau peuvent en principe être établis pour toute durée de référence et pour tout territoire. En pratique toutefois, le choix de l'une et de l'autre doit s'adapter aux possibilités de disposer des données nécessaires avec assez de validité ainsi qu'à la signification et à l'utilité des résultats comptables. Le champ le plus approprié est le bassin hydrographique assez grand pour rendre possible une correspondance avec un ensemble d'unités administratives. La durée de référence la mieux adaptée est l'année (civile ou hydrologique) ou la saison.

\section{III — UN INSTRUMENT D'ANALYSE ET D'INFORMATION}

Cette comptabilité analytique permet d'abord de réunir les chiffrages de tous les composants du bilan d'eau du système et de comparer leurs ordres de grandeur. Elle permet ensuite de comptabiliser les échanges d'eau intérieurs : soit spontanés (ou induits par les agents aménageurs ou exploitants) entre sous-systèmes de ressource, soit organisés entre agents, soit réalisés entre agents et sous-systèmes de ressource, dans les deux sens, donc les sources d'approvisionnement de chaque secteur et les pressions de ceux-ci sur les ressources. Elle permet encore d'expliquer les variations de stocks naturels ou artificiels au cours de la durée de référence du 


\begin{tabular}{|c|c|c|c|c|}
\hline \multicolumn{5}{|c|}{$\begin{array}{l}\text { ENTRÉES DANS LE SYSTĖME EAUX } \\
\text { CONTINENTALES }\end{array}$} \\
\hline $\begin{array}{c}\text { Précipita- } \\
\text { tions }\end{array}$ & $\begin{array}{c}\text { Ecoule- } \\
\text { ment } \\
\text { entrant }\end{array}$ & $\begin{array}{l}\text { Retours } \\
\text { d'eau }\end{array}$ & Irrigation & $\begin{array}{l}\text { Sous-total } \\
\text { Entrtes }\end{array}$ \\
\hline & & & & B \\
\hline 552,0 & & & 3,5 & 555. \\
\hline & $\mathcal{\varepsilon}$ & 7,0 & & 7. \\
\hline 20,5 & & & & 20,5 \\
\hline 1,5 & & 0,0 & & 1. \\
\hline 1,0 & 18,0 & 23,0 & & 42,0 \\
\hline 575,0 & 18,0 & 30. & 3,5 & 626. \\
\hline
\end{tabular}
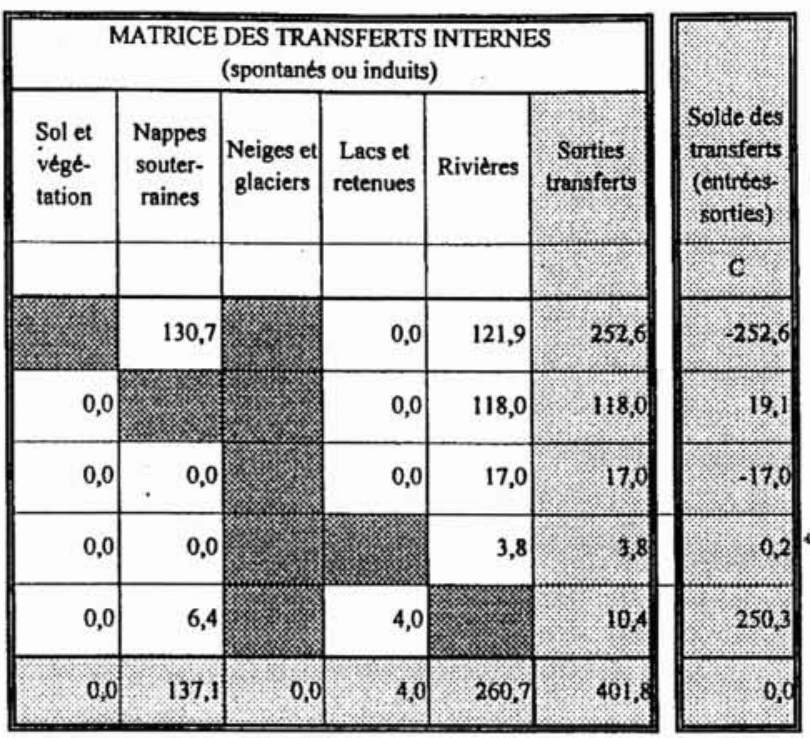

\begin{tabular}{|r|r|r|r|}
\hline \multicolumn{3}{|c|}{ SORTIES DU SYSTĖME EAUX } \\
CONTINENTALES
\end{tabular}

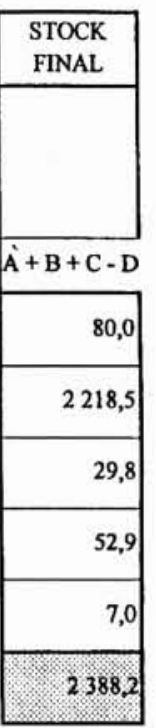

Tableau 2. Compte d'utilisations d'eau France entière - année 1981 en milliards de $m^{3}$

\begin{tabular}{|c|c|c|c|c|c|c|c|}
\hline \multirow{3}{*}{\multicolumn{2}{|c|}{ Operations: }} & \multicolumn{6}{|c|}{ ENTRÉES DANS LE SYSTĖME DUUTLISATION } \\
\hline & & \multicolumn{2}{|c|}{ Prelèvements } & \multirow[t]{2}{*}{$\begin{array}{l}\text { Impor- } \\
\text { tations }\end{array}$} & \multirow{2}{*}{$\begin{array}{c}\begin{array}{c}\text { Sous-total } \\
\text { Entrtes }\end{array} \\
A\end{array}$} & \multirow{2}{*}{$\begin{array}{c}\begin{array}{c}\text { Entress } \\
\text { Echan } \\
\text { 8es }\end{array} \\
8 \\
8\end{array}$} & \multirow{2}{*}{\begin{tabular}{|c|}
$\begin{array}{c}\text { Total } \\
\text { Envites }\end{array}$ \\
$\mathrm{A}+\mathrm{B}$ \\
\end{tabular}} \\
\hline & & $\begin{array}{l}\text { eaux } \\
\text { superf }\end{array}$ & $\begin{array}{l}\text { eaux } \\
\text { sout }\end{array}$ & & & & \\
\hline \multicolumn{2}{|l|}{\begin{tabular}{|l} 
Prod-distrib \\
d'eau potable
\end{tabular}} & 2,10 & $\begin{array}{l}1 \\
3,50\end{array}$ & 0,00 & 3,60 & 0,60 & 6,20 \\
\hline \multicolumn{2}{|l|}{$\begin{array}{l}\text { Prod-distrib } \\
\text { deau d'irrig. }\end{array}$} & 0,00 & 0,33 & 0,00 & 0,33 & 0,00 & 0,33 \\
\hline \multicolumn{2}{|l|}{$\begin{array}{l}\text { Entreprises } \\
\text { indus. \& mines }\end{array}$} & 3,47 & 1,77 & 0,00 & 5,24 & 0,82 & 6.06 \\
\hline \multicolumn{2}{|l|}{$\begin{array}{l}\text { Producteurs } \\
\text { d'ellectricité }\end{array}$} & 19,53 & $\varepsilon$ & 0,00 & 19,53 & 0.00 & 19,53 \\
\hline \multicolumn{2}{|l|}{ Agriculteurs } & 1,30 & 2,90 & 0,00 & 4,20 & 0,30 & 4,50 \\
\hline \multicolumn{2}{|l|}{ Ménages } & & 0,05 & 0,00 & 0,05 & 2,42 & 2,42 \\
\hline \multirow[t]{2}{*}{\begin{tabular}{|l} 
Services \\
publics
\end{tabular}} & Asst & 0,00 & 0,00 & 0,00 & 0,00 & 2,08 & 208 \\
\hline & Autres & 2,22 & $\varepsilon$ & $\varepsilon$ & 2,22 & 0,66 & 2,88 \\
\hline \multicolumn{2}{|l|}{ TOTAL: } & 28,62 & 8.55 & & 37,17 & 6,88 & 44,0 \\
\hline
\end{tabular}
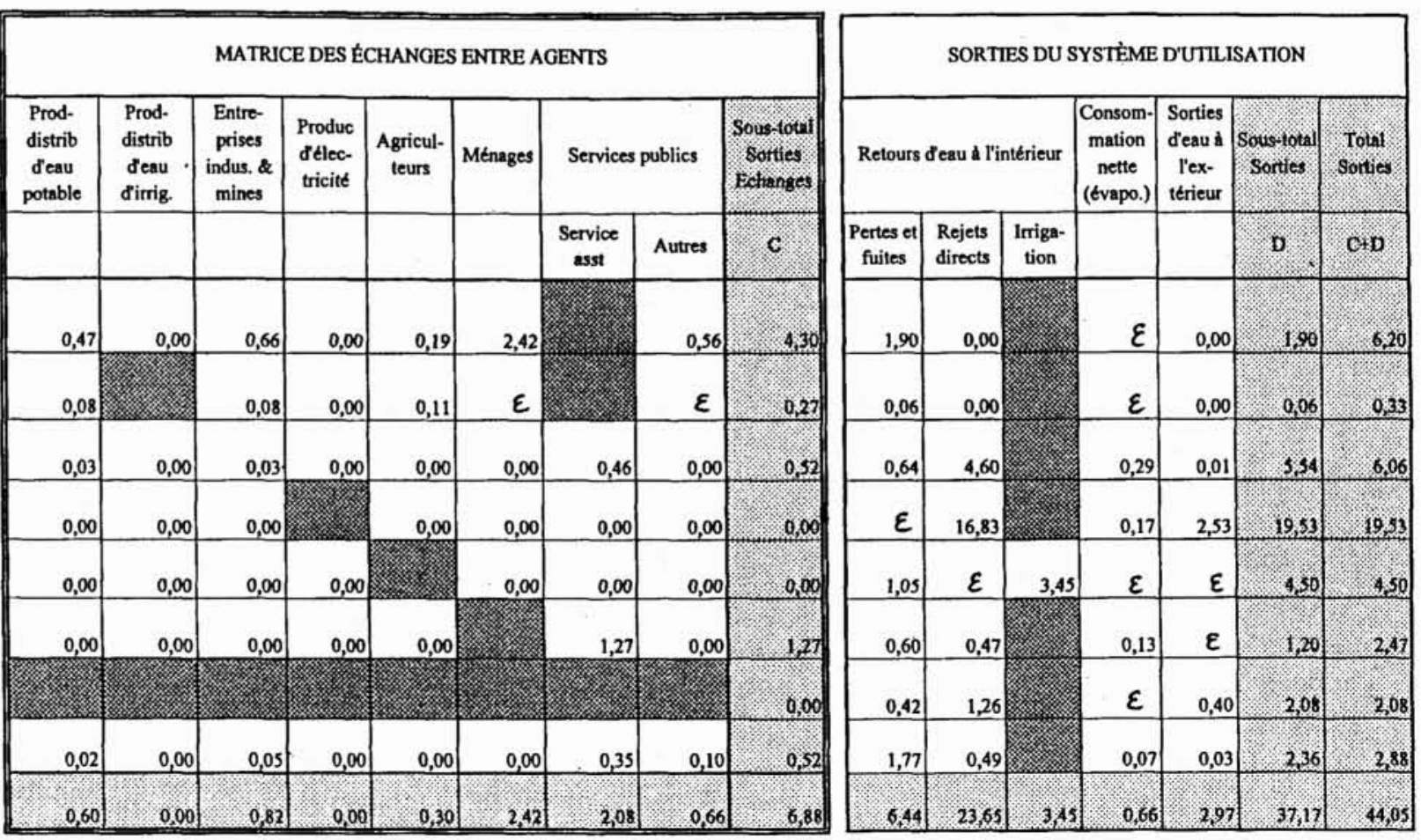
compte par les différences entre flux entrés et sortis. (cf l'exemple du compte de la France 1981, tableaux 1 et 2).

Les résultats comptables apportent des réponses à diverses demandes d'information sur le fonctionnement et l'état du système de ressource ainsi que sur les utilisations et leurs pressions quantitatives sur les ressources ( $\mathrm{cf}$ en encadré 1, les informations apportées par le compte de la France en 1981). En particulier ils facilitent le calcul de différents indicateurs appropriés pour l'analyse des situations et leur comparaison avec d'autres systèmes, ou avec le même système à d'autres périodes.

Enfin, cette comptabilité est applicable aux études prospectives en permettant de comparer les utilisations projetées à la ressource soit en moyenne (ou mieux suivant différentes fréquences d'occurrences d'apports), soit en projetant une distribution interannuelle antérieure conservant la même variabilité (cf les études de “ gestion prévisionnelle "), tout en prenant en compte le degré de régulation permis par les variations de stock.

\section{IV — EN PROJET : LES COMPTES DE L'EAU DES “ BASSINS RNDE ” EN FRANCE}

La comptabilité conjointe des ressources et des utilisations est un instrument de synthèse approprié des données rassemblées. Elle est envisagée par l'IFEN dans le cadre des " bassins RNDE ” (au nombre de 55) définis comme unités territoriales pour la gestion des données.

Cet affinement des références territoriales des comptes, associé à une subdivision des références temporelles, éprouvé dans l'exercice de l'IFEN sur la Haute-Loire [4] entraîne diverses conséquences sur les modalités d'établissement et sur la portée des comptes :

- sur la comptabilité des ressources : nécessités d'ajustement des données hydrologiques issues de la Banque Hydro (les bassins RNDE ne sont pas délimités en fonction de la localisation des stations de mesure), de définition conventionnelle des ressources externes (pour les bassins RNDE inscrits dans un grand bassin, à apports de l'amont simples ou multiples), d'estimation des variations de stock, notamment d'eau souterraine.

- sur la comptabilité des utilisations : nécessités d'adaptation des sources statistiques dont les références territoriales different de celles des ressources (données issues des déclarations de prélèvement et centralisées auprès des Agences de l'Eau ou d'autres organismes comme EDF...), de prise en compte de transferts inter-bassins dans certains cas (données EDF, VNF, compagnies d'aménagement...).

Un programme étagé dans le temps de réalisation des comptes de l'eau a été lancé : il comporte une première étape (fin 1999) de comptes simplifiés pour l'ensemble de la France et par bassins RNDE. La simplification porte sur la collecte et la prise en compte des prélèvements d'eau, abordés par voie statistique. En revanche, la ressource primaire (pluie, pluie efficace, écoulements) est modélisée en détail de manière à servir de source de données à la fois pour ces comptes et leurs développements ultérieurs, mais aussi pour des comptes de qualité, dont le calcul simplifié a été réalisé en 1998 (rapport en cours).

\section{BIBLIOGRAPHIE}

[1] Collectif (1986) Les comptes du patrimoine naturel (INSEE. les collections de I'INSEE 137-138 C, n 535-536, Déc. 552 p. Paris). Chap. 5, J. Margat, le compte des eaux continentales

[2] OCDE (1990) Etude pilote concernant les eaux intérieures. Compte des eaux continentales (OCDE, Comité de l'environnement, Groupe sur l'état de l'environnement, ENV/EC/SE (90) 24. Août, 25 p. Paris)

[3] Bauer A. et Costi A. (1993) Elaboration de comptes pilotes de I'eau dans le bassin du fleuve Brantas, Indonésie (Inst, pour une Politique Européenne de l'Environnement / DDCSTE, fév., 211 p. Paris)

[4] Belvaud E. (1995) Un nouvel outil de gestion de l'environnement : la comptabilité du patrimoine naturel (Univ. Nice Sophia Antipolis / Utah State Univ., mémoire de stage, sept, 41 p. + Annexes, Nice)

[5] Naredo J.M., Gasco J.M. (1994) Spanish Water Accounts (Spanish Minist. of Publics Works. Transport and the Environment. Summary Report, 25 p. + Annexes, Madrid)

[6] Babillot P. (1995) Compte de l'eau sur le bassin de la haute Loire (IFEN, juil., 126 p. Orléans)

[7] Babillot P. et Margat J. (1996) Ressources et utilisations de l'eau du bassin de la Loire. Essai de comptabilité régionale de l'eau (Colloque de la Soc. Hydrot. de France " La Loire ". Juin. Tours, Publ. La Houille Blanche $6 / 7, \mathrm{pp} .41-46$ )

[8] Germain C. (1997) Modélisation sous Nopolu System 2 des comptes de qualité par bassin versant par une méthode simplifiée, des pressions anthropiques d'origine agricole sur les bassins versants (IFEN / Univ. de Tours. rapport de stage, 50 p. + Annexes, Tours)

[9] Bauer A. (1993) Comptes de l'eau, guide méthodologique (Inst. pour une Politique Européenne de I'Environnement / DDCSTE, sept., 217 p. Paris)

110| Comolet A. (1990) La comptabilisation de la nature, éléments pour une analyse critique du système français de comptes du patrimoine naturel (Univ. Paris I, thèse de doctorat, fév, Paris)

[11] Margat J. (1987) Quand les eaux nous sont comptées. L'eau et les comptes du patrimoine naturel (Annales des Mines $n^{\circ} 10$, Oct., pp.84-87)

[12] Margat J., Weber J.L. (1988) Comptabilité des ressources naturelles. Propositions pour l'établissement de comptes des eaux continentales. (OCDE, Comité de l'environnement, Groupe sur l'état de l'environnement. Nov., ENV/SE/88.21, 18 p. Paris)

|13| Weber J.L. (1984) L'articulation des comptes du patrimoine naturel et de la comptabilité économique nationale. L'exemple du compte des " eaux continentales" (Conf. statisticiens européens, Paris 16-19 avril, INSEE, Doc de travail 6,35 p.) 